\title{
Effect of Sperm DNA Fragmentation on Embryo Quality in Normal Responder Women in In Vitro Fertilization and Intracytoplasmic Sperm Injection
}

\author{
Su Mi Kim ${ }^{1,2}$, Seul Ki Kim², ${ }^{2,3 y u n g ~ C h u l ~ J e e ~}{ }^{2,3}$, and Seok Hyun Kim² \\ ${ }^{1}$ Department of Obstetrics and Gynecology, Chungbuk National University Hospital, Cheongju; \\ ${ }^{2}$ Department of Obstetrics and Gynecology, Seoul National University College of Medicine, Seoul; \\ ${ }^{3}$ Department of Obstetrics and Gynecology, Seoul National University Bundang Hospital, Seongnam, Korea.
}

Purpose: To investigate the associations between sperm DNA fragmentation (SDF) and embryo formation rate in normal responder women to in vitro fertilization/intracytoplasmic sperm injection (IVF/ICSI).

Materials and Methods: Fifty-three consecutive, fresh IVF/ICSI cycles performed from 2014 to 2017 were selected. All women were normal responders (4 to 14 mature oocytes were retrieved) and at least one normally fertilized oocyte with two pronuclei was obtained in all cycles. Semen was collected on the day of oocyte retrieval, and SDF levels were measured by sperm chromatin dispersion test (Halosperm assay). At day 3 after insemination, embryo quality was evaluated by morphologic criteria and categorized as $\mathrm{A} / \mathrm{B} / \mathrm{C} / \mathrm{D}$. Top quality embryo were defined as grade $\mathrm{A}$ embryos with seven cells or more.

Results: SDF levels showed a positive linear correlation with the male's age $(\mathrm{r}=0.307, p=0.025)$ and a negative linear correlation with sperm motility ( $\mathrm{r}=-0.491, p<0.0001)$. To achieve top-quality or a grade A embryo formation rate $>70 \%$, the cut-off value SDF was $<30.7 \%$ for each. Among individuals with SDF $<30.7 \%$, the median top-quality or grade A embryo formation rate was significantly higher than that among individuals with SDF $\geq 30.7 \%$ ( $38.1 \%$ vs. $20.0 \%, p=0.038 ; 50 \%$ vs. $25.0 \%, p=0.017$ ).

Conclusion: In normal responder women, high SDF level resulted in low day 3 embryo formation rates. Our results suggest a paternal effect on embryo quality in IVF/ICSI cycles.

Key Words: In vitro fertilization, sperm DNA fragmentation, sperm chromatin dispersion test, embryo quality

\section{INTRODUCTION}

Sperm DNA integrity is essential for precise genetic transmission to a developing embryo and has been proposed as a biomarker for healthy sperm. Sperm DNA fragmentation (SDF) can be induced during chromatin remodeling in spermiogenesis, especially replacement of histone proteins by protamine. ${ }^{1}$

Received: November 29, 2018 Revised: February 22, 2019

Accepted: March 6, 2019

Corresponding author: Byung Chul Jee, MD, PhD, Department of Obstetrics and Gynecology, Seoul National University Bundang Hospital, 82 Gumi-ro 173beon-gil, Bundang-gu, Seongnam 13620, Korea

Tel: 82-31-787-7254, Fax: 82-31-787-4054, E-mail: blasto@snubh.org

-The authors have no potential conflicts of interest to disclose.

(C) Copyright: Yonsei University College of Medicine 2019

This is an Open Access article distributed under the terms of the Creative Commons Attribution Non-Commercial License (https://creativecommons.org/licenses/ by-nc/4.0) which permits unrestricted non-commercial use, distribution, and reproduction in any medium, provided the original work is properly cited.
Other causes of SDF included defective apoptosis, excessive reactive oxygen species, and environmental toxins. ${ }^{2}$ Although debatable, it has been reported that high SDF levels reduce clinical pregnancy rates ${ }^{3-7}$ and increase miscarriage rates in in vitro fertilization (IVF) cycles. ${ }^{8,9}$

Obtaining good-quality embryos is fundamental and leads to success in assisted reproduction. DNA-defective sperm in fertilization could potentially decrease embryo quality. However, evidence for this is insufficient. There are large discrepancies between published studies regarding the impact of SDF on embryo quality. ${ }^{10-16}$ Some researchers have suggested that there is a negative correlation between SDF and embryo quality, ${ }^{10-13}$ while others have found no association between them..$^{14-16}$

In the aforementioned studies, SDF was measured by different methods: terminal deoxynucleotidyl transferase dUTP nick-end labeling (TUNEL) assay, ${ }^{11,14}$ sperm chromatin structure assay, ${ }^{12,15}$ single cell gel electrophoresis (Comet) assay, ${ }^{10,13}$ 
or sperm chromatin dispersion (SCD) test. ${ }^{16}$ Among various methodologies, SCD test has been reported to be simple, less expensive, and highly reproducible. ${ }^{17}$

In previous studies, the majority of researchers selected women with various ovarian responses to gonadotropin, including poor responders. However, it appears to be reasonable to include normal responders only because poor responders are less likely to yield top-quality or good-quality embryos regardless of SDF. Two previous reports included normal responders only, ${ }^{11,14}$ and only Avendaño, et al. ${ }^{11}$ found a negative association between SDF level as measured by TUNEL assay and embryo quality.

To our knowledge, this is the first study to analyze correlations between SDF as measured by SCD test and embryo quality only in normal responders. The present study investigated the association of SDF level with three embryo formation rates at day 3 [per fertilized oocyte with two pronuclei (2PN)] in normal responder women.

\section{MATERIALS AND METHODS}

\section{Study population}

Fifty-three consecutive fresh IVF/intracytoplasmic sperm injection (ICSI) cycles were selected. All cycles were performed between 2014 and 2017 at Seoul National University Bundang Hospital. IVF/ICSI cycles were included if they were normal responders (i.e., 4 to 14 mature oocytes were retrieved) and at least one 2PN zygote was obtained. The median ages of women and their male partners were 35.0 (interquartile range: 32.0-39.5) and 38.0 (interquartile range: 35.0-44.0) years, respectively. Indications for IVF were unexplained $(\mathrm{n}=22)$, tubal $(n=12)$, male $(n=8)$, endometriosis $(n=5)$, uterine $(n=4)$, polycystic ovary syndrome $(n=1)$, and old age $(n=1)$. This retrospective study was approved by the Institutional Review Board of Seoul National University Bundang Hospital (IRB No. B-1806-474-106).

\section{Ovarian stimulation and oocyte retrieval}

Ovarian stimulation was performed with recombinant folliclestimulating hormone (FSH) (Gonal-F, Merck-Serono, Darmstadt, Germany; or Follitrope, LG Chem, Seoul, Korea), highly purified human menopausal gonadotrophin (Menopur, Ferring, Saint-Prex, Switzerland), or recombinant FSH/luteinizing hormone (Pergoveris, Merck-Serono). The dose of gonadotropin was adjusted individually based on follicular response monitored by transvaginal ultrasonography. Pituitary downregulation was achieved with a flexible GnRH antagonist protocol (Cetrotide $0.25 \mathrm{mg} / \mathrm{d}$, Merck-Serono) $(\mathrm{n}=49)$ or a midluteal long protocol using GnRH agonist (Decapeptyl $0.1 \mathrm{mg} /$ $\mathrm{d}$, Ferring) ( $\mathrm{n}=3$ ). Pituitary down-regulation was not employed in a single cycle. When the leading follicle reached a mean diameter of $\geq 18 \mathrm{~mm}$ or two follicles reached a mean diameter of $\geq 17 \mathrm{~mm}, 250 \mu \mathrm{g}$ of recombinant hCG (Ovidrel, Merck-Serono) was injected subcutaneously. Oocyte retrieval was performed at 35-36 hours after hCG injection. If immature oocytes were retrieved, they were cultured in in vitro maturation media. Once matured, they were counted as mature oocytes.

\section{Measurement of SDF, sperm preparation, and insemination}

Semen samples were collected on the day of oocyte retrieval. Standard sperm quality was assessed according to the World Health Organization guidelines (5th edition, 2010). ${ }^{18}$ Normal sperm was defined as semen volume $\geq 1.5 \mathrm{~mL}$, concentration $\geq 15 \times 10^{6} / \mathrm{mL}$, motility $\geq 40 \%$, and a percentage of normal form using strict criteria $\geq 4 \%$.

After one washing, SDF was measured by SCD test using Halosperm assay (Halotech DNA, Madrid, Spain), as previously described in our institutional protocol..$^{19}$ Briefly, an aliquot of the semen sample was diluted with Ham's F-10 medium (Sage, Trumbull, CT, USA) to $5 \times 10^{6} / \mathrm{mL}$ to $10 \times 10^{6} / \mathrm{mL}$. Agarose gel from the kit was placed in a water bath at $90-100^{\circ} \mathrm{C}$ for 5 minutes and then at $37^{\circ} \mathrm{C}$ for 5 minutes. This was followed by adding and mixing $25 \mu \mathrm{L}$ of semen in a marked Eppendorf test tube. The mixture of $15 \mu \mathrm{L}$ was placed on a super-coated slide and covered with a coverslip. Slides were then placed in a refrigerator at $4^{\circ} \mathrm{C}$ for 5 minutes to create a microgel with embedded sperm cells. After obtaining slides from the refrigerator, coverslips were gently removed, followed by immersing slides in acid denaturation solution ( $80 \mu \mathrm{L}$ in $10 \mathrm{~mL}$ of distilled water) for 7 minutes. These slides were then immersed in a lysis solution from the kit and incubated for 25 minutes. After washing with distilled water, slides were dehydrated in increasing concentrations of ethanol $(70,90$, and $100 \%)$ for 2 minutes and then air-dried. The slides were stained with Diff-quick solution I and II ( 7 minutes for each), rinsed with distilled water, and air-dried at room temperature.

Each slide was examined under a light microscopy at $\times 100$ magnification. At least 300 sperms were scored. Each sperm was classified as large halo, medium halo, small halo, no halo, or degraded. A large halo meant that the halo width was similar to or higher than the minor diameter of the core. A medium halo meant that the halo size was between large halo and small halo. A small halo meant that the halo width was similar to or smaller than one-third of the minor diameter of the core. Degraded sperm was similar to sperm with no halo but weakly or irregularly stained. SDF level (\%) was calculated as (the number of small halo+no halo+degraded form)/total sperm cell counted.

The remaining semen samples were processed by a discontinuous gradient (Sydney IVF Sperm Gradient, COOK Medial, Brisbane, Australia) as described in the kit instructions and our institutional protocol., ${ }^{8,19}$ Mature oocytes were inseminated by conventional methods ( 18 cycles), ICSI ( 29 cycles), or split insemination ( 6 cycles). Fertilization was confirmed by 
observing 2PN zygotes on day 1 after insemination.

\section{Embryo quality assessment}

Embryo quality was evaluated by morphological criteria based on the degree of fragmentation and the regularity of blastomeres on day 3 after insemination. Embryos were graded as follows: grade A, $0 \%$ anucleate fragments, regularity of blastomeres, no apparent morphological abnormality; grade $\mathrm{B},<20 \%$ anucleate fragments, regularity of blastomeres, no apparent morphological abnormality; grade C, 20-50\% anucleate fragments, irregularity of blastomeres, no apparent morphological abnormality; and grade D, >50\% anucleate fragments, irregularity of blastomeres, and apparent morphological abnormalities. ${ }^{20}$ The top-quality embryo was defined if they showed seven cells or more with grade A. Day 3 embryo formation rate was always calculated per 2PN zygote. In the present study, three embryo formation rates were employed: top-quality embryo formation rate, grade $\mathrm{A}$ embryo formation rate, and grade $\mathrm{A}$ or $\mathrm{B}$ embryo formation rate.

\section{Embryo transfer and IVF outcome measures}

The embryos were transferred at 3 days $(n=37)$ or 5 days $(n=$ 10) after oocyte retrieval. Serum hCG levels were determined at 14 days after oocyte retrieval. Those with a positive hCG result underwent transvaginal ultrasound to confirm an intrauterine pregnancy. Clinical pregnancy was defined as the presence of one or more gestational sacs with a fetal heart beat.

\section{Statistical analysis}

All statistical analyses were performed using SPSS 22 (IBM Corp., Armonk, NY, USA). Spearman correlation rank test was used to analyze the associations of SDF levels with standard sperm quality and three embryo formation rates. A receiver operating characteristics (ROC) curve analysis was used to determine the specific cut-off value of SDF to predict specific embryo formation rate. Mann-Whitney U test was used to compare parameters between groups. Proportions between groups were compared using Pearson's chi-square test or Fisher's exact test if the observed cell number was less than five. All $p$ values $<0.05$ were considered significant.

\section{RESULTS}

The interquartile range of SDF levels was $16.0 \%$ to $44.5 \%$ (median: $28.0 \%)$. SDF levels showed a positive linear correlation with the male's age $(\mathrm{r}=0.307, p=0.025)$ and a negative linear correlation with sperm motility $(\mathrm{r}=-0.491, p<0.0001)$. The estimated equation was as follows: SDF level $(\%)=16.786+[0.945 \times$ husband's age (yr)] - $[0.465 \times$ motility $(\%)](p<0.0001)$. SDF levels had no relationship with sperm concentration $(r=-0.07$, $p=0.614$ ) or the percentage of normal form ( $\mathrm{r}=-0.08, p=0.590)$.

The median value of top-quality embryo formation was $33.3 \%$ (interquartile range: 14.3-58.6\%): grade A embryo formation rate was $33.3 \%$ (interquartile range: $17.4-61.3 \%$ ), and grade $\mathrm{A}$ or B embryo formation rate was $75.0 \%$ (interquartile range: 50 $100 \%)$.

SDF levels exhibited a negative relationship with top-quality embryo formation ( $\mathrm{r}=-0.249, p=0.073$ ) and grade A embryo formation ( $\mathrm{r}=-0.240, p=0.083$ ), but did not reach statistical significance. SDF levels had no association with grade A or B embryo formation rate $(\mathrm{r}=-0.143, p=0.309)$.

ROC curve analysis revealed that the cut-off value of SDF was $<30.7 \%$ for prediction of a top-quality or grade A embryo formation rate $>70 \%$ with statistical significance (Table 1 ). This cut-off value had good sensitivity and an excellent negative predicted value. Among individuals with SDF $<30.7 \%$, the median top-quality embryo formation rate was significantly higher than that among individuals with SDF $\geq 30.7 \%$ (38.1\% vs. $20.0 \%, p=0.038$ ) (Table 2). Among individuals with SDF $<30.7 \%$, the median grade A embryo formation rate was also significantly higher than that among individuals with SDF $\geq 30.7 \%$ ( $50.0 \%$ vs. $25.0 \%, p=0.017$ ). Among those with SDF $<30.7 \%$, the clinical pregnancy rate was higher than that in subjects with $\mathrm{SDF} \geq 30.7 \%$, although the difference did not show statistical significance.

\section{DISCUSSION}

In the present study, SDF levels exhibited a negative impact with top-quality or grade A embryo formation rate in normal responder women. The cut-off value of SDF $<30.7 \%$ could predict top-quality or grade A embryo formation rate $>70 \%$ with a statistical significance. Between groups with SDF $<30.7 \%$ and $\mathrm{SDF} \geq 30.7 \%$, top-quality or grade A embryo formation rate was significantly different.

Analysis of sperm quality is based on standardized protocols recommended by the World Health Organization in 2010, ${ }^{18}$ although the conventional semen parameters do not reliably predict the outcomes of assisted reproductive technology. ${ }^{21}$ Male factor infertility is diagnosed by abnormal semen pa-

Table 1. Cut-Off Value of Sperm DNA Fragmentation to Predict Top-Quality or Grade A Embryo Formation Rate $>70 \%$ in Normal Responders

\begin{tabular}{lcc}
\hline & $\begin{array}{c}\text { Top quality embryo } \\
\text { formation rate }>\mathbf{7 0 \%}\end{array}$ & $\begin{array}{c}\text { Grade A embryo } \\
\text { formation rate }>\mathbf{7 0 \%}\end{array}$ \\
\hline Cut-off value (\%) & $<30.7$ & $<30.7$ \\
Area under the curve & 0.727 & 0.750 \\
95\% confidence interval & $0.554-0.900$ & $0.614-0.886$ \\
Sensitivity & 80.0 & 87.5 \\
Specificity & 54.2 & 51.1 \\
Positive likelihood ratio & 1.48 & 1.71 \\
Negative likelihood ratio & 0.44 & 0.26 \\
Positive predicted value & 13.3 & 23.3 \\
Negative predicted value & 95.7 & 95.7 \\
\hline
\end{tabular}


Table 2. Comparison of Cycle Characteristics according to the Cut-Off Value of SDF in Normal Responders

\begin{tabular}{|c|c|c|c|}
\hline & \multicolumn{2}{|c|}{ SDF level } & \multirow{2}{*}{$p$ value } \\
\hline & $<30.7 \%$ (30 cycles) & $\geq 30.7 \%$ ( 23 cycles $)$ & \\
\hline Age of female (yr) & $35[32,38.3]$ & $35[32,40]$ & 0.732 \\
\hline Age of male (yr) & $38[35,43]$ & $39[35,46]$ & 0.335 \\
\hline BMl of female $\left(\mathrm{kg} / \mathrm{m}^{2}\right)$ & $22.8[20.3,25.8]$ & $21.7[20.3,23.3]$ & 0.229 \\
\hline \multicolumn{4}{|l|}{ Causes of infertility, $\mathrm{n}(\%)$} \\
\hline Unexplained & $12(40.0)$ & $10(43.5)$ & \\
\hline Tubal & $11(36.7)$ & $1(4.3)$ & \\
\hline Male & $1(3.3)$ & $7(30.4)$ & \\
\hline Endometriosis & $3(10.0)$ & $2(8.7)$ & \\
\hline Uterine & $2(6.7)$ & $2(8.7)$ & \\
\hline Polycystic ovary syndrome & $1(3.3)$ & $0(0)$ & \\
\hline Old age & $0(0)$ & $1(4.3)$ & \\
\hline Serum anti-Mullerian hormone (ng/mL) & $2.89[1.77,6.60]$ & $2.62[1.16,6.76]$ & 0.663 \\
\hline Serum estradiol at triggering day $(\mathrm{pg} / \mathrm{mL})$ & $1032[676,1446]$ & $1996[847,2878]$ & 0.159 \\
\hline Dose of gonadotropin (IU) & $2100[1725,2400]$ & $1650[1350,2200]$ & 0.136 \\
\hline \multicolumn{4}{|l|}{ Sperm characteristics } \\
\hline Volume (mL) & $3[2,3.5]$ & $3[2.5,4.5]$ & 0.177 \\
\hline Concentration $\left(\times 10^{6} / \mathrm{mL}\right)$ & $100.5[43.4,168.5]$ & $66[30,178.9]$ & 0.346 \\
\hline Motility (\%) & $52.7[37.9,68.7]$ & $36.0[30.1,56.5]$ & 0.011 \\
\hline Total motile sperm $\left(\times 10^{6}\right)$ & $192[63,270]$ & $105[38,270]$ & 0.370 \\
\hline Normal form (\%) & $4.3[2.0,6.5]$ & $3.6[1.0,6.7]$ & 0.495 \\
\hline No. of previous cycles & $2[1,4]$ & $1[1,2]$ & 0.039 \\
\hline No. of mature oocytes retrieved & $6.5[4,10]$ & $7[5,9]$ & 0.959 \\
\hline \multicolumn{4}{|l|}{ Method of insemination, $\mathrm{n}(\%)$} \\
\hline Conventional & $12(40.0)$ & $6(26.1)$ & \\
\hline Intracytoplasmic sperm injection & $15(50.0)$ & $14(60.9)$ & \\
\hline Split insemination & $3(10.0)$ & $3(13.0)$ & \\
\hline Normal fertilization rate with two pronuclei (\%) & $80[71.4,100]$ & $77.8[70.0,100.0]$ & 0.600 \\
\hline No. of zygotes with two pronuclei & $6[4,7.3]$ & $6[4,7]$ & 0.771 \\
\hline No. of top-quality embryos at day 3 & $2[1,4]$ & $1[1,2]$ & 0.067 \\
\hline No. of grade A embryos at day 3 & $2.5[1,4]$ & $1[1,2]$ & 0.030 \\
\hline No. of grade A or B embryos at day 3 & $4[3,6]$ & $4[2,5]$ & 0.146 \\
\hline Top-quality embryo formation rate (\%) & $38.1[25.0,62.5]$ & $20.0[12.5,50.0]$ & 0.038 \\
\hline Grade A embryo formation rate (\%) & $50.0[25.0,67.5]$ & $25.0[12.5,50.0]$ & 0.017 \\
\hline Grade A or B embryo formation rate (\%) & $80.0[55.4,100.0]$ & $62.5[45.5,100.0]$ & 0.230 \\
\hline \multicolumn{4}{|l|}{ No. of ET cycles, n } \\
\hline At day 3 & 22 & 15 & \\
\hline At day 5 & 6 & 4 & \\
\hline \multicolumn{4}{|l|}{ Clinical pregnancy rate per transfer, \% (n) } \\
\hline Day 3 ET & $27.3(6 / 27)$ & $20.0(3 / 20)$ & 0.711 \\
\hline Day 5 ET & $50.0(3 / 6)$ & $25.0(1 / 4)$ & 0.571 \\
\hline
\end{tabular}

SDF, sperm DNA fragmentation; BMI, body mass index; ET, embryo transfer.

Data are presented as a median [interquartile range] unless otherwise noticed. The median values were compared by Mann-Whitney U test. The proportions were compared by the chi-square test or the Fisher's exact test.

rameters, but could be present even if the semen analysis is normal. Agarwal and Allamaneni ${ }^{22}$ reported that $15 \%$ of men with infertility problems were classified with normozoospermia. Therefore, researchers have investigated other markers to predict male infertility in a more clinically useful manner. An increasing number of studies suggest that SDF could be a po- tential biomarker of semen quality. ${ }^{23}$ Moreover, it is well established that the completion of fertilization process and subsequent embryo development depends, in part, on the integrity of the sperm DNA. ${ }^{16}$

In order to investigate exactly how SDF affects embryo quality, it is appropriate to control the number of retrieved oocytes. 
In a recent meta-analysis, there was a strong positive association between the number of oocytes collected and the number of top- or good-quality embryos at day $2 / 3(\mathrm{r}=0.791, p<0.001){ }^{24}$ Considering the quantitative aspects, poor responders with few oocytes have a lower chance to form top-quality or good-quality embryos regardless of SDF. In our study, we selected only normal responders for more convincing evidence that SDF deteriorates embryo quality.

It has been reported that oocytes have the capability to repair damaged DNA of sperm in a murine model. ${ }^{25,26}$ Although oocytes can repair damaged DNA of sperm, it seems to have a threshold. Beyond such threshold, damaged sperm DNA appears unrepairable $\mathrm{e}^{12,27}$ and may negatively impact embryos. It was traditionally accepted that the male genome becomes activated at the later developmental stage of embryogenesis. Interestingly, high SDF levels could influence early embryo development up to the 4-cell stage, in which the paternal genome is thought to be inactive. ${ }^{13}$ SDF may activate additional DNA repair pathways, and the embryo development could be delayed, resulting in poor embryo quality. ${ }^{28}$

In the aforementioned studies that reported a negative correlation between SDF and embryo quality, ${ }^{10-13}$ there were no definite inclusion criteria regarding ovarian responses, except the study conducted by Avendaño, et al., ${ }^{11}$ which included women with more than four oocytes collected. Avendaño, et al. ${ }^{11}$ evaluated embryo quality using mean embryo score, which may not accurately reflect the embryo quality because it uses mean values and because it is difficult to score the morula or embryo with compaction at day 3. Even if embryo formation rate was employed, ${ }^{10,12,13}$ using the total number of embryos for the denominator could be affected by the number of oocytes. It seems more suitable to use normally fertilized oocytes with 2PN for the denominator.

In this study, we found that the cut-off value of SDF was $<30.7 \%$ to predict a top-quality or grade A embryo formation rate $>70 \%$. Our cut-off value of SDF was similar to that of a previous report, in which a cut-off value of SDF $<27 \%$ was significantly associated with higher top-quality embryo formation rates at day $3 .^{12}$ Interestingly, cut-off values of SDF measured with SCD test to obtain a high pregnancy rate have been reported to be $30-35 \% .^{29}$

The main strengths of this study were including only normal responders, using $2 \mathrm{PN}$ for the denominator of embryo formation rate, and determining SDF in the actual sperm sample used for IVF. The limitations of this study were the retrospective nature of the work. We could not analyze the study population by dividing it into conventional IVF or ICSI because of the small sample size. Further studies with large sample sizes are required to exclude other possible confounding factors, such as a women's age.

In conclusion, our results indicated that SDF levels $<30.7 \%$ could be a good predictor to achieve a top-quality or grade A embryo formation rate $>70 \%$. Our results suggest a paternal effect on embryo quality in IVF cycles even in normal responder women.

\section{AUTHOR CONTRIBUTIONS}

Conceptualization: Byung Chul Jee. Data curation: Byung Chul Jee. Formal analysis: Su Mi Kim. Investigation: Su Mi Kim. Methodology: Seul Ki Kim. Project administration: Seok Hyun Kim. Resources: Seul Ki Kim. Software: Su Mi Kim. Supervision; Byung Chul Jee, Seok Hyun Kim. Validation: Seul Ki Kim. Visualization: Su Mi Kim. Writing_original draft: Su Mi Kim. Writing_review \& editing: Byung Chul Jee, Seul Ki Kim.

\section{ORCID iDs}

$\begin{array}{ll}\text { Su Mi Kim } & \text { https://orcid.org/0000-0002-5236-7184 } \\ \text { Seul Ki Kim } & \text { https://orcid.org/0000-0002-1647-6711 } \\ \text { Byung Chul Jee } & \text { https://orcid.org/0000-0003-2289-6090 } \\ \text { Seok Hyun Kim } & \text { https://orcid.org/0000-0003-0649-3224 }\end{array}$

\section{REFERENCES}

1. Santiso R, Tamayo M, Gosálvez J, Meseguer M, Garrido N, Fernández JL. Simultaneous determination in situ of DNA fragmentation and 8-oxoguanine in human sperm. Fertil Steril 2010;93:314-8.

2. Panner Selvam MK, Agarwal A. A systematic review on sperm DNA fragmentation in male factor infertility: laboratory assessment. Arab J Urol 2018;16:65-76.

3. Collins JA, Barnhart KT, Schlegel PN. Do sperm DNA integrity tests predict pregnancy with in vitro fertilization? Fertil Steril 2008;89: 823-31.

4. Li Z, Wang L, Cai J, Huang H. Correlation of sperm DNA damage with IVF and ICSI outcomes: a systematic review and meta-analysis. J Assist Reprod Genet 2006;23:367-76.

5. Simon L, Emery BR, Carrell DT. Review: diagnosis and impact of sperm DNA alterations in assisted reproduction. Best Pract Res Clin Obstet Gynaecol 2017;44:38-56.

6. Zhang Z, Zhu L, Jiang H, Chen H, Chen Y, Dai Y. Sperm DNA fragmentation index and pregnancy outcome after IVF or ICSI: a meta-analysis. J Assist Reprod Genet 2015;32:17-26.

7. Zhao J, Zhang Q, Wang Y, Li Y. Whether sperm deoxyribonucleic acid fragmentation has an effect on pregnancy and miscarriage after in vitro fertilization/intracytoplasmic sperm injection: a systematic review and meta-analysis. Fertil Steril 2014;102:998-1005.

8. Choi HY, Kim SK, Kim SH, Choi YM, Jee BC. Impact of sperm DNA fragmentation on clinical in vitro fertilization outcomes. Clin Exp Reprod Med 2017;44:224-31.

9. Robinson L, Gallos ID, Conner SJ, Rajkhowa M, Miller D, Lewis S, et al. The effect of sperm DNA fragmentation on miscarriage rates: a systematic review and meta-analysis. Hum Reprod 2012;27: 2908-17.

10. Tomsu M, Sharma V, Miller D. Embryo quality and IVF treatment outcomes may correlate with different sperm comet assay parameters. Hum Reprod 2002;17:1856-62.

11. Avendaño C, Franchi A, Duran H, Oehninger S. DNA fragmentation of normal spermatozoa negatively impacts embryo quality and intracytoplasmic sperm injection outcome. Fertil Steril 2010; 94:549-57.

12. Niu ZH, Shi HJ, Zhang HQ, Zhang AJ, Sun YJ, Feng Y. Sperm chromatin structure assay results after swim-up are related only to embryo quality but not to fertilization and pregnancy rates following IVF. Asian J Androl 2011;13:862-6. 
13. Simon L, Murphy K, Shamsi MB, Liu L, Emery B, Aston KI, et al. Paternal influence of sperm DNA integrity on early embryonic development. Hum Reprod 2014;29:2402-12.

14. Frydman N, Prisant N, Hesters L, Frydman R, Tachdjian G, CohenBacrie $\mathrm{P}$, et al. Adequate ovarian follicular status does not prevent the decrease in pregnancy rates associated with high sperm DNA fragmentation. Fertil Steril 2008;89:92-7.

15. Lin MH, Kuo-Kuang Lee R, Li SH, Lu CH, Sun FJ, Hwu YM. Sperm chromatin structure assay parameters are not related to fertilization rates, embryo quality, and pregnancy rates in in vitro fertilization and intracytoplasmic sperm injection, but might be related to spontaneous abortion rates. Fertil Steril 2008;90:352-9.

16. Anifandis G, Bounartzi T, Messini CI, Dafopoulos K, Markandona $\mathrm{R}$, Sotiriou S, et al. Sperm DNA fragmentation measured by Halosperm does not impact on embryo quality and ongoing pregnancy rates in IVF/ICSI treatments. Andrologia 2015;47:295-302.

17. Fernández JL, Muriel L, Goyanes V, Segrelles E, Gosálvez J, Enciso $\mathrm{M}$, et al. Simple determination of human sperm DNA fragmentation with an improved sperm chromatin dispersion test. Fertil Steril 2005;84:833-42.

18. World Health Organization. WHO laboratory manual for the examination and processing of human semen. 5th ed. Geneva: World Health Organization; 2010.

19. Kim SW, Jee BC, Kim SK, Kim SH. Sperm DNA fragmentation and sex chromosome aneuploidy after swim-up versus density gradient centrifugation. Clin Exp Reprod Med 2017;44:201-6.

20. Bolton VN, Hawes SM, Taylor CT, Parsons JH. Development of spare human preimplantation embryos in vitro: an analysis of the correlations among gross morphology, cleavage rates, and development to the blastocyst. J In Vitro Fert Embryo Transf 1989;6:30-5.
21. Practice Committee of the American Society for Reproductive Medicine. The clinical utility of sperm DNA integrity testing: a guideline. Fertil Steril 2013;99:673-7.

22. Agarwal A, Allamaneni SS. The effect of sperm DNA damage on assisted reproduction outcomes. A review. Minerva Ginecol 2004;56: 235-45.

23. Agarwal A, Said TM. Role of sperm chromatin abnormalities and DNA damage in male infertility. Hum Reprod Update 2003;9:33145.

24. D'Hooghe T, Vermey B, Chua S, Zafarmand H, Wang R, Longobardi $\mathrm{S}$, et al. Is the number of oocytes aspirated after ovarian stimulation for IVF/ICSI associated with the number of top/good quality embryos? A systematic review and meta-analysis. Fertil Steril 2018;110:e206.

25. Ahmadi A, Ng SC. Developmental capacity of damaged spermatozoa. Hum Reprod 1999;14:2279-85.

26. Ahmadi A, Ng SC. Fertilizing ability of DNA-damaged spermatozoa. J Exp Zool 1999;284:696-704.

27. Fatehi AN, Bevers MM, Schoevers E, Roelen BA, Colenbrander B, Gadella BM. DNA damage in bovine sperm does not block fertilization and early embryonic development but induces apoptosis after the first cleavages. J Androl 2006;27:176-88.

28. Bazrgar M, Gourabi H, Yazdi PE, Vazirinasab H, Fakhri M, Hassani F, et al. DNA repair signalling pathway genes are overexpressed in poor-quality pre-implantation human embryos with complex aneuploidy. Eur J Obstet Gynecol Reprod Biol 2014;175:152-6.

29. Cissen M, Wely MV, Scholten I, Mansell S, Bruin JP, Mol BW, et al. Measuring sperm DNA fragmentation and clinical outcomes of medically assisted reproduction: a systematic review and metaanalysis. PLoS One 2016;11:e0165125. 